\title{
RELACIÓN ENTRE EL ÍNDICE DE CONTROL DE LA CORRUPCIÓN Y ALGUNAS VARIABLES SOCIALES, ECONÓMICAS E INSTITUCIONALES
}

\author{
José Enrique Báez Gómez \\ Universidad de Málaga
}

http://dx.doi.org/10.5209/rev_NOMA.2013.v38.42911

\begin{abstract}
Resumen.- La corrupción es actualmente una de las principales preocupaciones de los españoles y uno de los mayores problemas a nivel mundial. En este artículo se analizan los efectos perjudiciales de la corrupción sobre determinadas variables sociales, económicas e institucionales. Para ello, como medida de la corrupción se ha usado el Índice de Control de la Corrupción (uno de los seis Indicadores de Gobernabilidad desarrollados por el Banco Mundial), y como enfoque teórico se ha adoptado la Nueva Economía Institucional, un campo de la Economía que aunque es relativamente reciente ha adquirido importancia e interés en los últimos años.
\end{abstract}

Palabras clave.- índice de control de la corrupción, nueva economía institucional

\section{Relationship between the Index of Control of Corruption and some social, economic and institutional variables}

\begin{abstract}
Corruption is currently one of the main concerns of Spanish and one of the major problems of the world. This article discusses the harmful effects of corruption in certain social, economic and institutional variables. For this, the Index of Control of Corruption (one of the six Indicators of Governance developed by the World Bank) has been used as measure of corruption, and the New Institutional Economics (a field of Economics that is relatively recent but that has gained importance and interest in recent years) has been adopted as theoretical approach.
\end{abstract}

Keywords.- index of control of corruption, new institutional economics

\section{INTRODUCCIÓN}

La corrupción es un tema que desgraciadamente está de actualidad en nuestro país. Tal es la gravedad del asunto que en los últimos Barómetros del CIS (los correspondientes a febrero, marzo, abril y junio de 2013) la corrupción aparece como el segundo problema principal para los españoles, sólo por detrás del paro. Sin duda, los últimos casos de corrupción aparecidos en prensa, donde presuntamente están implicados altos cargos políticos y grandes personalidades del país, ha contribuido a ello. Pero la corrupción no es algo característico únicamente de España; en palabras de Cobus de Swardt, Director Ejecutivo de Transparencia Internacional, "la corrupción es el problema mundial del que más se habla". Precisamente, esta organización no gubernamental publica anualmente el Índice de Percepción de Corrupción, cuya escala va de 0 (percepción de altos niveles de corrupción) a 100 (percepción de bajos niveles de corrupción); en 2012, dos tercios de los 176 
países clasificados en este índice obtuvieron una puntuación inferior a $50^{1}$, lo cual muestra que queda todavía mucho por hacer en esta materia.

Además del Índice de Percepción de Corrupción elaborado por Transparencia Internacional, otra medida muy utilizada para medir la corrupción en los países es el Índice de Control de la Corrupción, que es uno de los seis Indicadores de Gobernabilidad desarrollados por el Banco Mundial, y que es el que se va a emplear en este artículo. No es casualidad que el Banco Mundial se preocupe por la corrupción y haya elaborado un índice como éste, ya que según este organismo los actos de corrupción equivalen al 5\% del PIB mundial ${ }^{2}$ y constituyen el obstáculo más grande al desarrollo económico y social, al debilitar el Estado de derecho, la libre competencia empresarial y el acceso a los servicios básicos. Y es que según los últimos datos de las Encuestas de Empresas publicados también por el Banco Mundial, a nivel mundial el promedio de empresas que hacen pagos informales para que "las cosas se hagan" es del $24,8 \%$, porcentaje que en países como Siria, Guinea o Bangladesh supera el $80 \%^{3}$.

\section{MARCO TEÓRICO}

El Índice de Control de la Corrupción mide, en una escala comprendida entre 2,5 y 2,5 (donde $-2,5$ representa el peor desempeño y 2,5 el mejor), el grado en que se ejerce el poder público en beneficio privado, incluidas las modalidades de corrupción en pequeña y gran escala, y el control del Estado por minorías selectas. En el Anexo se muestra la puntuación que cada país obtuvo en este índice en 2011. No obstante, aunque este índice sólo se refiere a la corrupción en el ámbito público, hay que apuntar que la corrupción también se da en la esfera privada, si bien es cierto que "las definiciones de corrupción predominantes limitan el fenómeno, injustificada y engañosamente, al sector público" (Hodgson y Jiang, 2008:56).

Uno de los colectivos que comete este sesgo es el de los economistas, ya que son muchos los autores que cuando hablan de corrupción únicamente se refirieren a la corrupción pública. Por ejemplo, Jain (2001:73) señala que "aunque es difícil estar de acuerdo en una definición precisa, hay consenso en que la corrupción se refiere a actos en los que el poder del cargo público es usado para beneficio personal de una manera que contraviene las reglas del juego"; en Hodgson y Jiang (2008:58-59) se citan otros ejemplos, no sólo de economistas, sino también de otros científicos sociales.

Pero centrándonos en los economistas, ¿qué es lo que les lleva a limitar la corrupción únicamente al sector público? Una explicación podría encontrarse en la ideología neoliberal y su influencia en la corriente económica dominante (la Economía neoclásica), que tanto cuestiona el papel del Estado. Si definimos la corrupción como algo que sucede en el ámbito público y adoptamos este enfoque neoliberal, ello nos incita a pensar que la solución al problema de la corrupción es la reducción del tamaño del Estado, que es precisamente lo que

1

http://www.transparency.org/news/pressrelease/20121205_comunicado_de_prensa_indice_de_ percepcion_de_la_corrupcion_2012

2 http://gatintelligence.com/index.php/es/gat-prensa/276-rrhh-digital

${ }^{3}$ http://espanol.enterprisesurveys.org/Data/ExploreTopics/corruption 
propugna el Neoliberalismo. En este sentido, autores como Savas (2000:223) y Torres (2000:145) proponen la privatización como vía para reducir la corrupción, y el Premio Nobel Gary Becker llegó incluso a declarar que "si abolimos el Estado, abolimos la corrupción". Sin embargo, Rose-Ackerman (1996) advierte que si no se maneja con cuidado la reducción del Estado, los estímulos para la corrupción pueden aumentar en antiguas áreas y surgir en nuevas, ya que el proceso de privatización está lleno de oportunidades de corrupción. Por otro lado, Acemoglu y Verdier (2000:196) argumentan que en muchos casos, ante un mal funcionamiento de los mercados, la intervención gubernamental es necesaria aun cuando encierre el peligro de corrupción, ya que en estos casos la corrupción puede ser el menor de los males. Además, la evidencia no muestra que los niveles de corrupción estén relacionados estrechamente con el tamaño del Estado; de hecho, algunos de los países menos corruptos según los indicadores publicados, como es el caso de Dinamarca, Finlandia, Holanda, Noruega y Suecia, tienen los mayores niveles de gasto público en proporción al PIB.

Y ya que hablamos de Economía, ¿cómo explica ésta la corrupción? Si usamos la teoría económica ortodoxa, la corrupción se explicaría en términos de racionalidad del individuo, maximización de su utilidad y análisis costebeneficio: si la probabilidad de que se descubra un acto corrupto y/o su castigo es bajo, entonces es rentable ser corrupto. Siguiendo esta lógica, si suponemos que para una determinada acción corrupta los costes (castigos) son relativamente bajos y son los mismos para todo el mundo, entonces todo individuo que pudiera realizar dicho acto corrupto lo llevaría a cabo. Esta suposición queda desmontada, por ejemplo, con un estudio de Fisman y Miguel (2007) en el que se analiza la propensión de los diplomáticos extranjeros a saltarse las normas de estacionamiento de la ciudad de Nueva York, y en el cual descubren que el número de violaciones en el estacionamiento de los diplomáticos está fuertemente correlacionado con las acciones de corrupción existentes en su país de origen; estos resultados sugieren que las normas culturales o sociales relacionadas con la corrupción están por lo visto profundamente arraigadas, y que por tanto hay otros factores diferentes a la aplicación de la ley que son determinantes importantes de un comportamiento corrupto. Esos otros factores podrían ser explicados por la Economía Institucional, ya que, "debido al énfasis en las instituciones, los economistas institucionalistas están más atentos a las reglas y a su transgresión" (Hodgson y Jiang, 2008:66); de hecho, Boehm (2005:248) sostiene que "un enfoque neoinstitucional del problema de la corrupción parece la metodología más adecuada para su análisis". A continuación se exponen brevemente algunos aspectos de este campo de la Economía que, pese al predominio de la teoría económica neoclásica, ha ido adquiriendo importancia, interés y reconocimiento en los últimos años; prueba de ello es la concesión del Premio Nobel de Economía a Ronald Coase (1991), Douglass North (1993), Oliver Williamson (2009) y Elinor Ostrom (2009), autores de la denominada Nueva Economía Institucional (NEI).

Para empezar, se muestran en la Tabla 1 las principales diferencias entre la Economía neoclásica y la Nueva Economía Institucional. 
Tabla 1. Principales diferencias entre la Economía neoclásica y la Nueva Economía Institucional

\begin{tabular}{|l|l|l|}
\cline { 2 - 3 } \multicolumn{1}{l|}{} & \multicolumn{1}{c|}{ Economía neoclásica } & Nueva Economía Institucional \\
\hline La Economía & Ciencia de la elección & Ciencia de la transacción \\
\hline Mercados & Instrumental & Limitada \\
\hline Costes de transacción & Eficientes & Imperfectos, con fricciones \\
\hline Instituciones & Ausencia de ellas & Positivos \\
\hline Empresa, ley y política & Son como cajas negras & $\begin{array}{l}\text { Son entendidas como reglas de } \\
\text { juego }\end{array}$ \\
\hline El tiemponstitucionales
\end{tabular}

FUENTE: Elaboración propia a partir de Caballero, G. (2011). "Economía de las instituciones: de Coase y North a Williamson y Ostrom". Ekonomiaz, 77, pp. 33-34.

Si a este enfoque institucionalista de la Economía se le coloca delante el adjetivo de "nueva" es porque antes hubo una teoría económica institucionalista "vieja". Así pues, hay dos vertientes del pensamiento económico acerca de las instituciones: la Vieja Economía Institucional ${ }^{4}$ y la Nueva Economía Institucional. Contrariamente a lo que podría pensarse, la NEI no emerge desde el viejo institucionalismo económico; es más, "el propio Coase (1984) afirmó que el trabajo de los viejos institucionalistas americanos no condujo a nada y que si los institucionalistas modernos tienen algún antecedente no hay que buscarlo en sus inmediatos predecesores" (Caballero, 2011:35). En la siguiente tabla se señalan las diferencias básicas entre estas dos vertientes.

\footnotetext{
${ }^{4}$ Parada (2003) prefiere denominarla Economía Institucional Original (EIO) para evitar el prejuicio de un "institucionalismo viejo o fuera de moda" frente a uno "nuevo o moderno".
} 
Tabla 2. Diferencias básicas entre el viejo y el nuevo institucionalismo económico

\begin{tabular}{|c|c|c|}
\hline & Vieja Economía Institucional & Nueva Economía Institucional \\
\hline $\begin{array}{l}\text { Relación con la } \\
\text { Economía neoclásica }\end{array}$ & Rechaza sus bases & Emerge de ella \\
\hline Enfoque que asume & Holista o sistémico & Individualismo metodológico \\
\hline Distribución/eficiencia & $\begin{array}{l}\text { Las cuestiones distributivas son } \\
\text { clave }\end{array}$ & $\begin{array}{l}\text { Criterio de eficiencia para el } \\
\text { análisis }\end{array}$ \\
\hline $\begin{array}{l}\text { Cómo es el } \\
\text { intercambio }\end{array}$ & $\begin{array}{l}\text { Relevancia de la coerción y el } \\
\text { poder }\end{array}$ & Libre entre iguales \\
\hline $\begin{array}{l}\text { Preferencias de los } \\
\text { individuos }\end{array}$ & No deben tomarse como dadas & Se toman como dadas \\
\hline $\begin{array}{l}\text { Comportamiento de } \\
\text { los individuos }\end{array}$ & Los hábitos son claves & Racional (racionalidad limitada) \\
\hline Instituciones como & Hábitos comunes de pensamiento & Reglas del juego \\
\hline $\begin{array}{l}\text { Perspectiva que } \\
\text { asume }\end{array}$ & $\begin{array}{l}\text { Conductista, colectivista y lejana al } \\
\text { formalismo }\end{array}$ & $\begin{array}{l}\text { Es más reduccionista, } \\
\text { individualista y formalista }\end{array}$ \\
\hline Principales autores & $\begin{array}{l}\text { Veblen, Commons, Ayres y } \\
\text { Mitchell }\end{array}$ & $\begin{array}{l}\text { Coase, North, Williamson y } \\
\text { Ostrom }\end{array}$ \\
\hline
\end{tabular}

FUENTE: Elaboración propia a partir de Caballero, G., op. cit., pp. 34-35

Además, los economistas de la NEI tienen una visión más favorable de los mercados y una visión más negativa del gobierno, aunque según Parada (2003), esto no significa que los economistas de la Vieja Economía Institucional sean más intervencionistas que los de la NEI. A pesar de estas diferencias, en los últimos años han aparecido trabajos en los que se observa un cierto acercamiento entre ambas vertientes; ello está en concordancia con el carácter interdisciplinar que adopta la NEI, buscando la colaboración mutua de la economía con otras ciencias sociales.

Centrándonos en la $\mathrm{NEI}$, su objeto de estudio son las instituciones y cómo éstas afectan a la economía y a la sociedad, aspectos que como hemos comentado anteriormente no han sido tenidos en cuenta por la teoría económica convencional (al menos hasta hace bien poco). Para North (1991), las instituciones son las reglas de juego que determinan las restricciones y los incentivos en la interacción económica, política y social; esas reglas pueden ser informales (como las tradiciones, los tabúes, las creencias y los códigos de conducta) o formales (constituciones, leyes, contratos, etc.). La importancia de las instituciones radica en que son el determinante subyacente del desempeño de las economías, ya que determinan los incentivos para invertir e innovar, así como los costes de transacción, dando forma a la dirección del cambio económico hacia el crecimiento, el estancamiento o el declive ${ }^{5}$.

${ }^{5}$ El propio Banco Mundial, en su estudio "Beyond the Washington Consensus: Institutions Matter" (1998), expone que las instituciones deben ser incorporadas al análisis de las realidades económicas y el diseño de políticas. 
Siguiendo a Bandeira (2009), los trabajos que intentan demostrar que las instituciones son la causa fundamental del desarrollo económico pueden dividirse en dos grupos: los que argumentan que las instituciones económicas formales que protegen los derechos de propiedad privada son las principales responsables del crecimiento económico, y los que argumentan que el factor clave para el desarrollo económico son las instituciones políticas que promueven el "buen gobierno". Relacionado con esto último, y aludiendo al modelo de Estado depredador planteado por North (aquél que se enriquece aún cuando implique el fracaso económico del país), en este artículo se analiza la relación entre el "mal gobierno" (centrándonos exclusivamente en la faceta corrupta de éste) y una serie de variables.

Son numerosos los trabajos donde se evidencian los efectos perjudiciales de la corrupción para los países; así, se ha comprobado que la corrupción desalienta la inversión privada (Mauro, 1995; Del Monte y Pagagni, 2001), altera el gasto del Gobierno disminuyendo los destinados a educación (Mauro, 1997), reduce la eficiencia de los gastos en inversión pública (Del Monte y Pagagni, 2001), limita el desarrollo de pequeñas y medianas empresas (Tanzi y Davoodi, 2002), entorpece la actividad innovadora (Varsekelis, 2006) e incide negativamente sobre el desarrollo humano (Amate y Guarnido, 2011).

\section{METODOLOGÍA}

Tras la revisión bibliográfica realizada, se quiere ver si existe relación lineal entre la corrupción (medida a través del Índice de Control de la Corrupción) y algunas variables sociales, económicas e institucionales, las cuales se detallan en la siguiente tabla:

Tabla 3. Descripción de las variables utilizadas

\begin{tabular}{|l|l|c|}
\hline \multicolumn{1}{|c|}{ Variable } & \multicolumn{1}{|c|}{ Descripción } & $\begin{array}{c}\text { Países } \\
\text { para los } \\
\text { que hay } \\
\text { datos }\end{array}$ \\
\hline PIBpc & PIB per cápita (US\$ a precios actuales) & 177 \\
\hline FBKf & Formación bruta de capital fijo (\% del PIB) & 144 \\
\hline Desempleo & Tasa de desempleo (\% de la población activa total) & 78 \\
\hline Deuda & Deuda del gobierno central (\% del PIB) & 54 \\
\hline Inflación & Inflación, precios al consumidor (\% anual) & 170 \\
\hline Gastosalud & Gasto total (público más privado) en salud (\% del PIB) & 184 \\
\hline Mortalidadinfantil & Tasa de mortalidad en los menores de 5 años (\%) & 161 \\
\hline Créditosprivado & Crédito interno al sector privado (\% del PIB) & 180 \\
\hline Tiemponegocio & Tiempo necesario para iniciar un negocio (días) & 180 \\
\hline Procedimientos & No de procedimientos iniciales para registrar una empresa & 180 \\
\hline Noimpuestos & Cantidad total de impuestos que pagan las empresas & \\
\hline & $\begin{array}{l}\text { Índice de desempeño logístico. Refleja las percepciones de la } \\
\text { logística de un país basadas en la eficiencia del proceso del } \\
\text { despacho de aduana, la calidad de } \\
\text { relacionada con el comercio y el transporte, la facilidad de }\end{array}$ & \\
\hline
\end{tabular}




\begin{tabular}{|c|c|c|}
\hline Logística & $\begin{array}{l}\text { acordar embarques a precios competitivos, la calidad de los } \\
\text { servicios logísticos, la capacidad de seguir y rastrear los } \\
\text { envíos, y la frecuencia con la cual los embarques llegan al } \\
\text { consignatario en el tiempo programado. Varía entre } 1 \text { (bajo } \\
\text { desempeño) y } 5 \text { (alto) }\end{array}$ & 153 \\
\hline Recaudación & Recaudación impositiva (\% del PIB) & 100 \\
\hline CréditosFMI & Uso de créditos del FMI (\% del PIB) & 122 \\
\hline Derechoslegales & $\begin{array}{l}\text { Índice de fortaleza de los derechos legales. Mide el grado en } \\
\text { el que las leyes de garantía y quiebra protegen los derechos } \\
\text { de los prestatarios y prestamistas y, de ese modo, facilitan el } \\
\text { otorgamiento de préstamos. Va de } 0 \text { (débil) a } 10 \text { (fuerte) }\end{array}$ & 180 \\
\hline Facilidadnegocios & $\begin{array}{l}\text { Índice de facilidad para hacer negocios. Clasifica los países } \\
\text { del } 1 \text { (reglamentaciones más favorables para los negocios) en } \\
\text { adelante }\end{array}$ & 180 \\
\hline Tiempoimpuestos & $\begin{array}{l}\text { Tiempo para preparar y pagar impuestos, en horas por año, } \\
\text { que lleva la preparación, la presentación y el pago (o } \\
\text { retención) de tres grandes tipos de impuestos: el impuesto } \\
\text { sobre las utilidades, el impuesto al valor agregado o a las } \\
\text { ventas, y los impuestos laborales, que incluyen los impuestos } \\
\text { sobre nóminas y las contribuciones a la seguridad social. }\end{array}$ & 180 \\
\hline Escañosmujeres & \begin{tabular}{|l} 
Proporción de escaños ocupados por mujeres en los \\
parlamentos nacionales (\%)
\end{tabular} & 180 \\
\hline IDH & Índice de Desarrollo Humano & 187 \\
\hline Estadoderecho & $\begin{array}{l}\text { Índice de Estado de Derecho. Mide el grado de confianza de } \\
\text { los agentes en las reglas sociales y su nivel de acatamiento, } \\
\text { incluidos la calidad del cumplimiento de los derechos de } \\
\text { propiedad, la policía y los tribunales, así como el riesgo de } \\
\text { que se cometan delitos. Varía entre }-2,5 \text { (el peor } \\
\text { desempeño) y } 2,5 \text { (el mejor). }\end{array}$ & 187 \\
\hline Corrupción & $\begin{array}{l}\text { Índice de Control de la Corrupción. Mide el grado en que se } \\
\text { ejerce el poder público en beneficio privado, incluidas las } \\
\text { modalidades de corrupción en pequeña y gran escala, y el } \\
\text { control del Estado por minorías selectas. Está comprendido } \\
\text { también entre }-2,5 \text { (el peor desempeño) y } 2,5 \text { (el mejor). }\end{array}$ & 187 \\
\hline
\end{tabular}

FUENTE: Elaboración propia

Todos los datos relativos a estas variables se refieren al año 2011 (excepto el Índice de desempeño logístico, que se refiere a 2010), y todos se han obtenido de la web del Banco Mundial ${ }^{6}$ (a excepción del IDH, cuyos datos se han obtenido de la web del Programa de las Naciones Unidas para el Desarrollo) ${ }^{7}$.

Para analizar la relación lineal entre el Índice de Control de la Corrupción y el resto de variables se va a realizar un análisis de correlaciones, así como un análisis gráfico de aquellas relaciones más significativas.

\footnotetext{
${ }^{6} \mathrm{http}: / /$ datos.bancomundial.org/indicador y http://info.worldbank.org/governance/wgi/index.asp

${ }^{7}$ http://hdrstats.undp.org/es/indicadores/103106.html
} 


\section{RESULTADOS}

A continuación se muestran los resultados del análisis de correlaciones:

Tabla 4. Correlación lineal entre el Índice de Control de la Corrupción y el resto de variables

\begin{tabular}{|l|c|l|c|}
\hline \multicolumn{1}{|c|}{ Variable } & Correlación de Pearson & \multicolumn{1}{c|}{ Variable } & Correlación de Pearson \\
\hline PIBpc & $0,747^{\star \star}$ & Noimpuestos & $-0,440^{\star \star}$ \\
\hline FBKf & $-0,118$ & Logística & $0,755^{\star \star}$ \\
\hline Desempleo & $-0,141$ & Recaudación & $0,365^{\star \star}$ \\
\hline Deuda & $0,370^{\star \star}$ & CréditosFMI & $-0,060$ \\
\hline Inflación & $-0,368^{\star \star}$ & Derechoslegales & $0,363^{\star \star}$ \\
\hline Gastosalud & $0,341^{\star \star}$ & Facilidadnegocios & $-0,741^{\star \star}$ \\
\hline Mortalidadinfantil & $-0,547^{\star \star}$ & Tiempoimpuestos & $-0,300^{\star \star}$ \\
\hline Créditosprivado & $0,712^{\star \star}$ & Escañosmujeres & $0,291^{\star \star}$ \\
\hline Tiemponegocio & $-0,193^{\star \star}$ & IDH & $0,698^{\star \star}$ \\
\hline Procedimientos & $-0,371^{\star \star}$ & Estadoderecho & $0,944^{\star \star}$ \\
\hline
\end{tabular}

** La correlación es significativa al nivel 0,01 (bilateral)

FUENTE: Elaboración propia

Como se observa en la tabla anterior, el Índice de Control de la Corrupción tiene una correlación lineal significativa con todas las variables seleccionadas (excepto con la formación bruta de capital fijo, la tasa de desempleo y el uso de créditos del FMI).

La correlación es negativa con las siguientes variables: Inflación, Mortalidadinfantil, Tiemponegocio, Procedimientos, Nimpuestos, Facilidadnegocios y Tiempoimpuestos, lo que significa que cuanto menor es el Índice de Control de la Corrupción ( $y$, por tanto, mayor corrupción hay), mayor es la inflación, mayor es la tasa de mortalidad en los menores de 5 años, más días son necesarios para iniciar un negocio, más procedimientos iniciales se necesitan para registrar una empresa, más cantidad de impuestos pagan las empresas, más desfavorables son las reglamentaciones para los negocios, y más horas al año se dedican para preparar y pagar impuestos.

Por el contrario, la correlación es positiva con las variables PIBpc, Deuda, Gastosalud, Créditosprivado, Logística, Recaudación, Derechoslegales, Escañosmujeres, IDH y Estadoderecho, lo cual significa que cuanto menor es el Índice de Control de la Corrupción ( $y$, por tanto, mayor corrupción hay), menor es la deuda del gobierno central, el gasto total en salud, el crédito interno al sector privado y la Recaudación impositiva (todo ello expresado como $\%$ del PIB), más bajo es el PIB per cápita, menor es el desempeño logístico, más débiles son las leyes de garantía y quiebra que protegen los derechos de los prestatarios y prestamistas, hay menos proporción de mujeres ocupando escaños en los parlamentos nacionales, más baja es la puntuación en el IDH, y menor es el grado de confianza en el Estado de derecho.

Una vez analizada la correlación lineal entre la corrupción y el resto de variables, se presentan los gráficos de dispersión entre el Índice de Control de la Corrupción y aquellas variables que muestran un coeficiente de correlación 
elevado con dicho índice, a saber: PIB per cápita, Crédito interno al sector privado (\% del PIB), Índice de desempeño logístico, Índice de facilidad para hacer negocios, Índice de Desarrollo Humano e Índice de Estado de Derecho. Tal como está definido el Índice de Control de la Corrupción (cuanto mayor es su valor, menos corrupción hay en el país), todas las relaciones directas (positivas) que se muestran a continuación tenemos que interpretarlas como inversas (negativas) en términos de corrupción, y viceversa.

Gráfico 1. Índice de Control de la Corrupción y PIB per cápita [FUENTE: Elaboración propia]

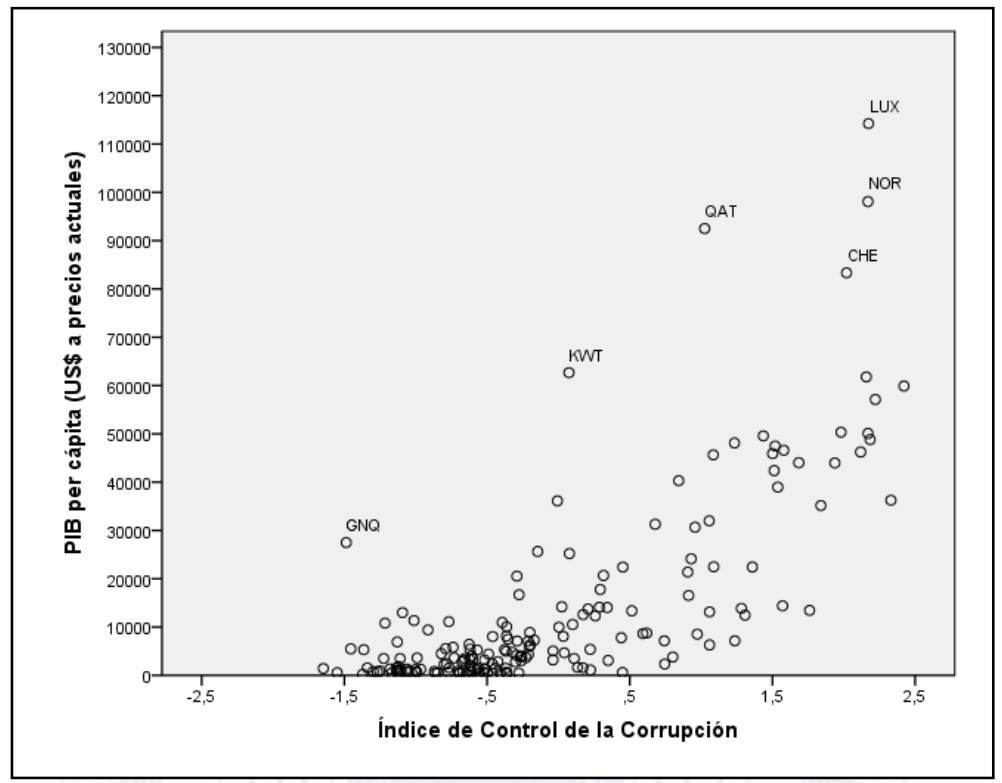

Como se puede observar, existe una relación negativa entre corrupción y PIB per cápita, de modo que los países pobres son más corruptos que los ricos. Esta relación ya fue verificada empíricamente en trabajos como los de Treisman (2000) o Alcaide y Larrú (2007), quienes encontraron una asociación estadística muy fuerte entre la corrupción y el ingreso per cápita.

Gráfico 2. Índice de Control de la Corrupción y Crédito interno al sector privado (\% del PIB) [FUENTE: Elaboración propia]

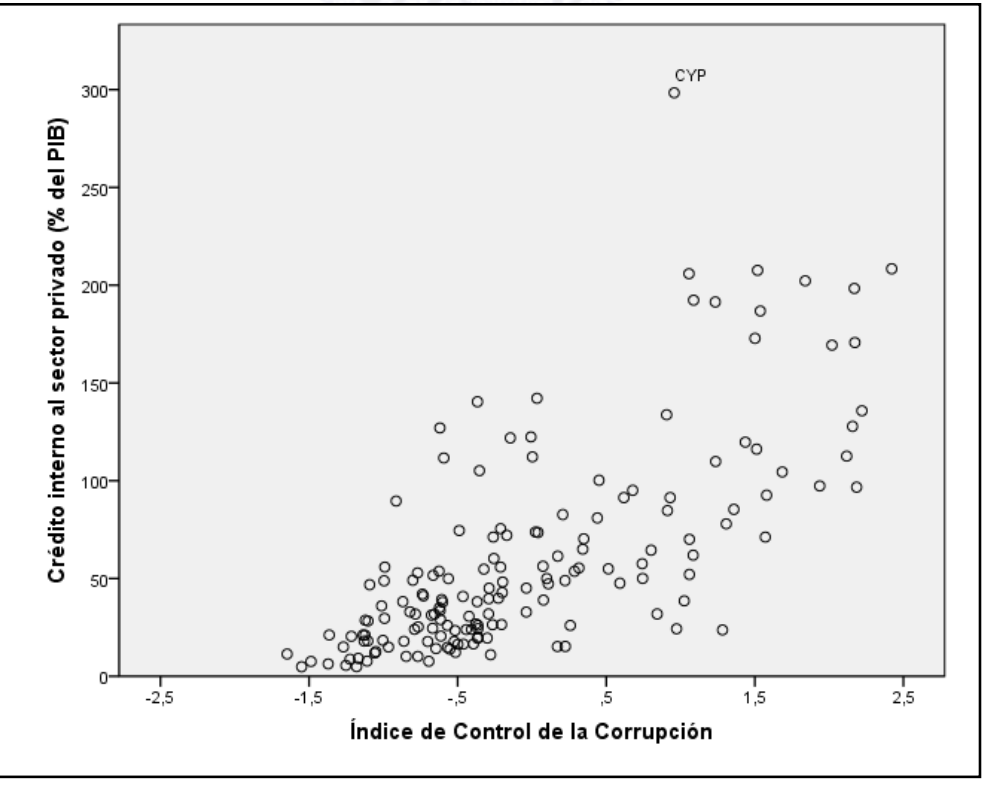


El gráfico 2 nos muestra una relación negativa entre corrupción y crédito interno al sector privado (\% PIB), de manera que cuanto mayor es la corrupción en un país menos recursos financieros se otorgan a sus empresas, lo cual dificulta la inversión y el desarrollo de éstas, aspectos que aparecen recogidos en los trabajos de Mauro (1995), Del Monte y Pagani (2001), Tanzi y Davoodi (2002).

Gráfico 3. Índice de Control de la Corrupción e Índice de desempeño logístico [FUENTE: Elaboración propia]

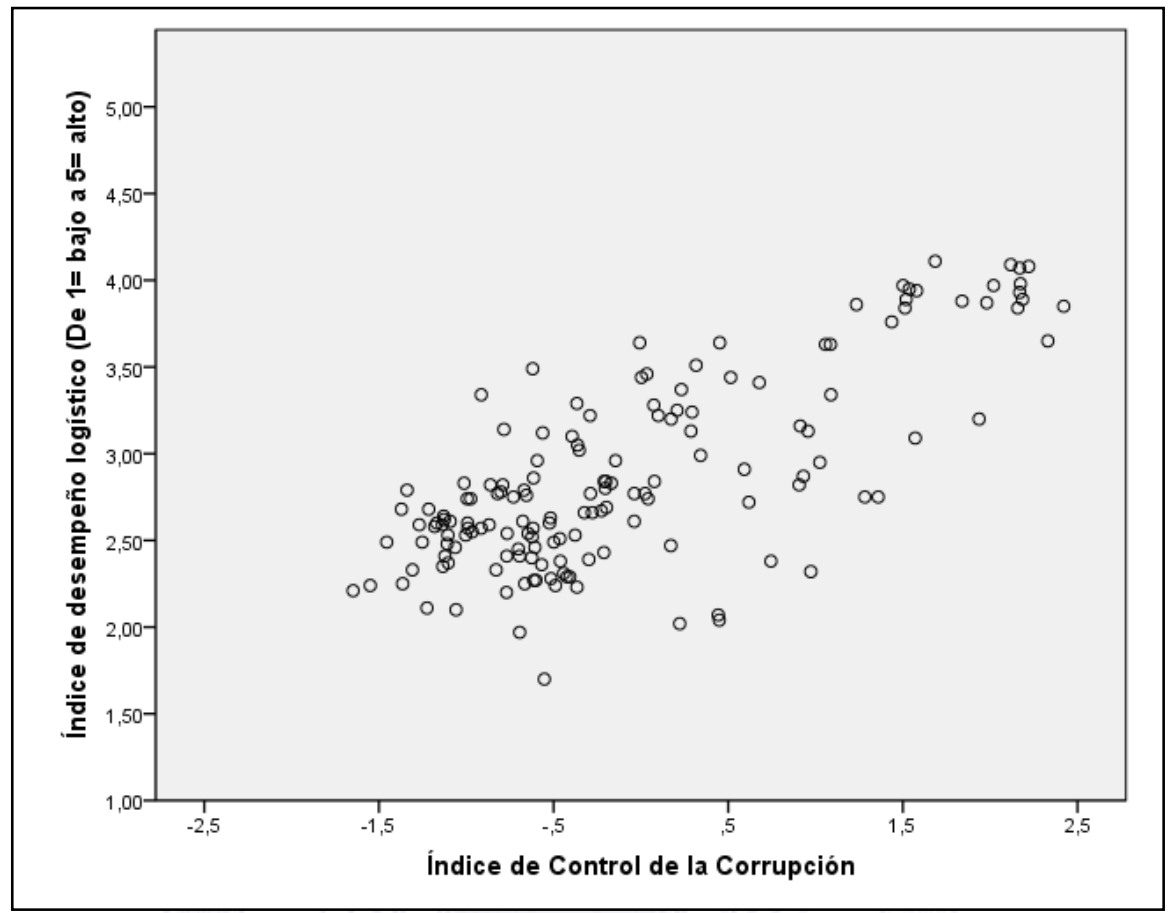

En el gráfico 3 nos encontramos de nuevo con una variable relacionada negativamente con la corrupción: el Índice de desempeño logístico. Así pues, cuanta más corrupción hay, peores son las percepciones de la logística de un país. Esto era algo de esperar, ya que uno de los aspectos que recoge el Índice de desempeño logístico es la eficiencia del proceso del despacho de aduana, y son numerosos los casos de corrupción descubiertos en las aduanas, sobre todo en ciertos países de América Latina (aunque también se han dado algunos recientemente en España) ${ }^{8}$.

8 Véanse por ejemplo las siguientes noticias aparecidas en prensa: http://elpais.com/diario/2008/09/23/espana/1222120816_850215.html

http://www.laprovincia.es/canarias/2012/02/13/quince-imputados-trama-corrupcionaduana/438291.html 
Gráfico 4. Índice de Control de la Corrupción e Índice de facilidad para hacer

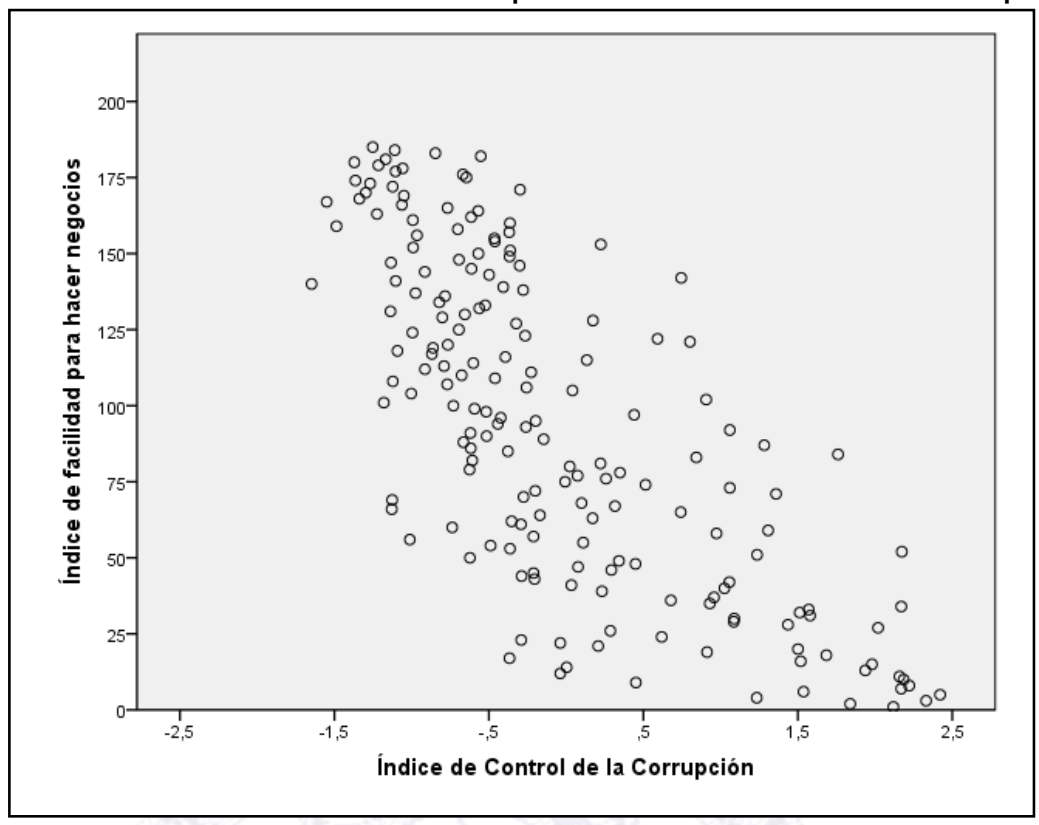

FUENTE: Elaboración propia

En el gráfico 4 se puede comprobar la existencia de una relación positiva entre corrupción y el Índice de facilidad para hacer negocios, que tal como está definido éste último implica que cuanta más corrupción hay en un país más desfavorables son sus reglamentaciones para los negocios. Esto tiene su lógica, pues si las leyes de un país dificultan la realización de negocios en él, la persona que realmente quiera hacer negocios en dicho país tratará de buscar alguna vía (como por ejemplo, corromper a funcionarios públicos) para conseguirlo.

Gráfico 5. Índice de Control de la Corrupción e Índice de Desarrollo Humano

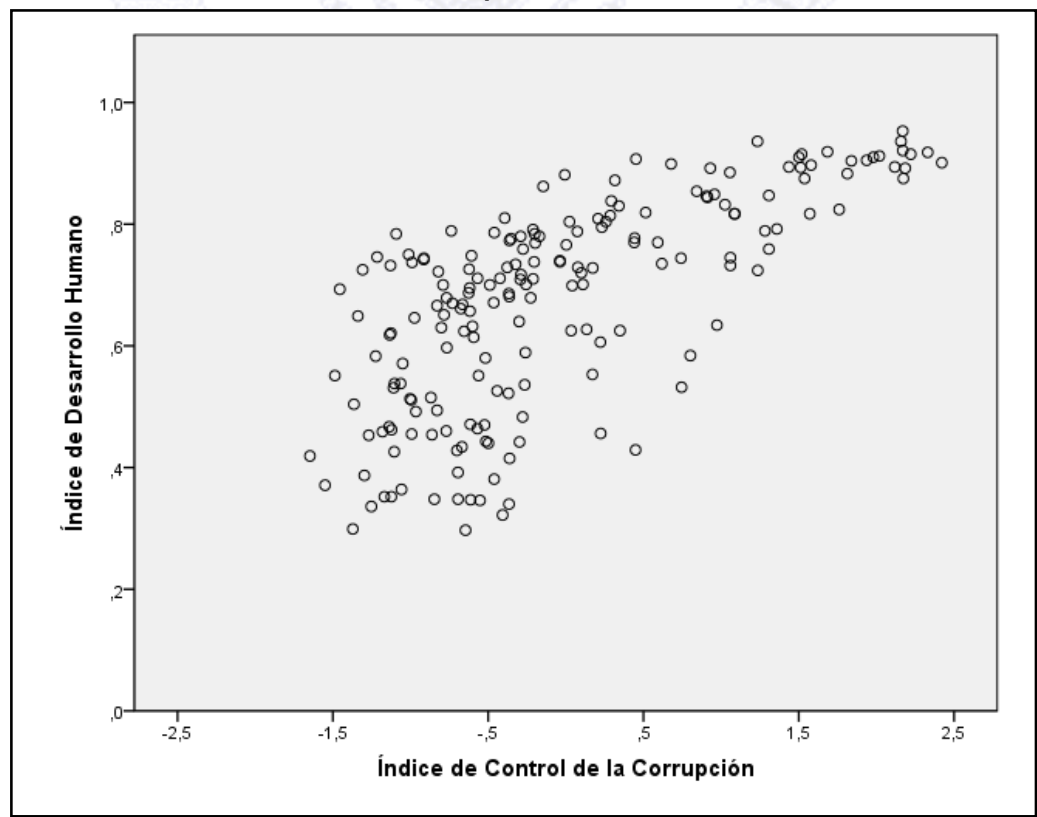

FUENTE: Elaboración propia 
Del gráfico 5 se desprende que existe una relación negativa entre corrupción e IDH, de modo que la corrupción afecta negativamente al desarrollo humano. Esta relación ha sido comprobada estadísticamente en investigaciones recientes como las de Alcaide y Larrú (2007) o Amate y Guarnido (2011).

Gráfico 6. Índice de Control de la Corrupción e Índice de Estado de Derecho

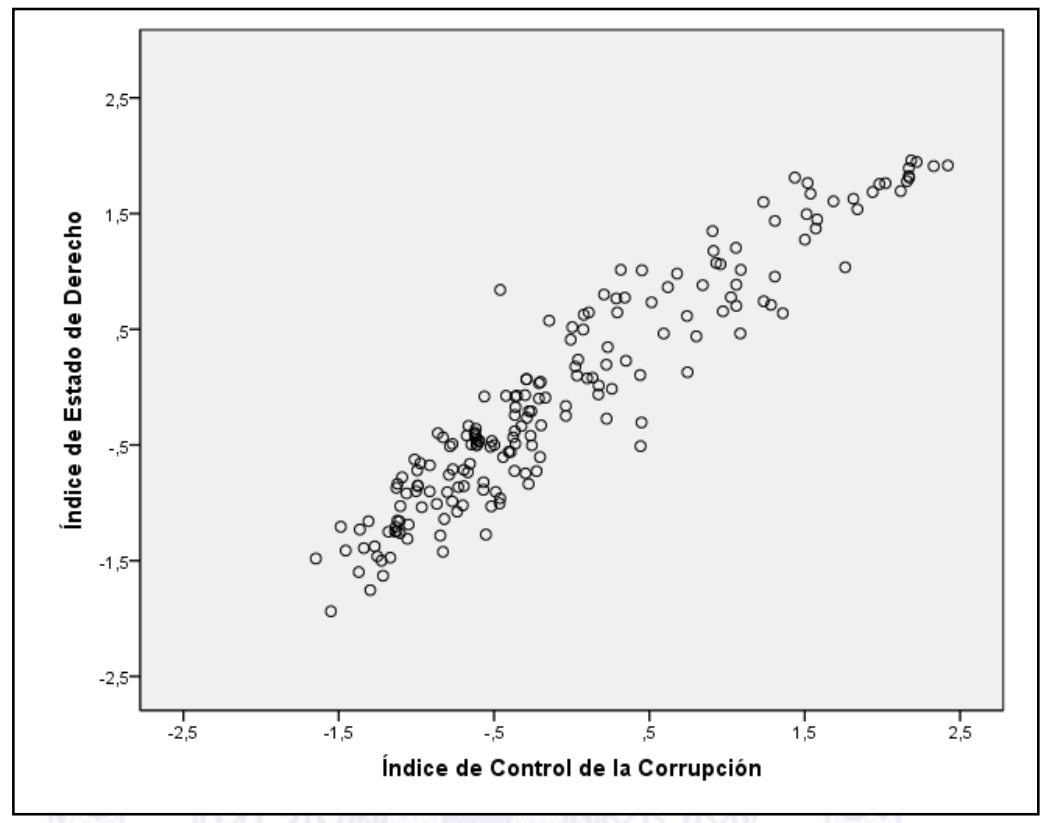

FUENTE: Elaboración propia

Para terminar, en el gráfico 6 se ve claramente una relación negativa entre corrupción y el Índice de Estado de Derecho, lo cual implica que cuanto mayor es la corrupción en un país menor es el grado de confianza de los agentes en las reglas sociales y su nivel de acatamiento, haciendo más difícil con ello el imperio de la ley. Así pues, la corrupción (sobre todo si se da en el ámbito judicial) socava el Estado de Derecho, ya que "cuando las cortes ceden ante la corrupción por avaricia o conveniencia política, la balanza de la justicia se inclina y el ciudadano común se ve perjudicado"9, lo cual provoca el descontento y la desconfianza de la ciudadanía hacia las instituciones.

\section{CONCLUSIONES}

La corrupción es un problema que sufren muchos países del mundo y al cual éstos deben poner remedio, dados los efectos negativos que tiene sobre la economía, los ciudadanos y las propias instituciones de dichos Estados.

\footnotetext{
9 Palabras de Huguette Labelle, Presidenta de Transparency International, durante la presentación del Informe Global de la Corrupción 2007. http://www.transparencia.org.es/INDICES_GLOBAL_DE_LA_CORRUPCI\%C3\%93N/INFORME \%20GLOBAL\%202007/Comunicado\%20de\%20Prensa\%20GCR\%202007.pdf
} 
Precisamente, las instituciones juegan un doble papel en este ámbito, ya que por un lado se ven afectadas por la corrupción, mientras que por otro son determinantes importantes de los comportamientos corruptos: "si el marco institucional premia la piratería, surgirán entonces organizaciones piratas" ${ }^{10}$.

Así pues, dada la importancia de las instituciones en este aspecto y la falta de respuestas convincentes por parte de la teoría económica convencional, en el artículo se ha adoptado como enfoque para el estudio de la corrupción la Nueva Economía Institucional.

Tras el análisis de correlaciones realizado entre el Índice de Control de la Corrupción y una serie de variables sociales, económicas e institucionales (apoyado con varios gráficos de dispersión), hemos obtenido unos resultados que confirman los efectos perjudiciales de la corrupción. En concreto, hemos comprobado que cuanto mayor es el nivel de corrupción de un país menor es su PIB per cápita, menos recursos financieros se otorgan a sus empresas, peores son las percepciones de la logística de ese país, más desfavorables son sus reglamentaciones para los negocios, más baja es su puntuación en el IDH, y menor es el grado de confianza de sus ciudadanos en el Estado de Derecho.

Como vemos, la corrupción no es para nada un asunto menor, ya que afecta a la vida de las personas, de las empresas y del propio Estado. Al hilo de esto último que he comentado, quisiera concluir con una cita de López Calera (1997), tomada de Nieto (2003:93), que bien podría extrapolarse por ejemplo a la situación española actual: "la corrupción política, más aún cuando llega a ser mera delincuencia común, está promoviendo una crisis de legitimidad en el Estado social y democrático de Derecho; de esa corrupción política provienen muchas de las críticas al Estado democrático; las gentes se quejan -y con razón- de los políticos, pero terminan quejándose del Estado a quienes esos políticos dicen representar".

\section{REFERENCIAS BIBLIOGRÁFICAS}

(2000) ACEMOGLU, Daron y VERDIER, Thierry, "The Choice between Market Failures and Corruption", The American Economic Review, 90(1), ISSN 0002-8282, Nashville, pp 194-211 <http://conferences.wcfia.harvard.edu/files/gov2126/files/acemoglumktfailureaer2000.pdf>.

(2007) ALCAIDE ZUGAZA, Laura y LARRÚ RAMOS, José María, "Corrupción, ayuda al desarrollo, pobreza y desarrollo humano", Boletín Económico de ICE, 2917, ISSN 0214-8307, Madrid, pp 37-58 <http://www.revistasice.com/cachepdf/BICE_2917_3758__A2F59B400B4112681D7AD09655B1FDF6.pdf>

(2011) AMATE FORTES, Ignacio y GUARNIDO RUEDA, Almudena, Factores determinantes del desarrollo económico y social, Ed. Servicio de Publicaciones de la Fundación Unicaja, Málaga.

${ }^{10}$ Palabras pronunciadas por Douglass C. North en Estocolmo el 9 de diciembre de 1993, en la conferencia dada al recibir el Premio Nobel de Economía http://www.eumed.net/cursecon/textos/north-nobel.htm 
(2009) BANDEIRA, Pablo, "Instituciones y desarrollo económico. Un marco conceptual". Revista de Economía Institucional, 20, ISSN 0124-5996, Bogotá, pp 355-373 <http://www.scielo.org.co/pdf/rei/v11n20/v11n20a13.pdf>

(2005) BOEHM, Frédéric, "Corrupción y captura en la regulación de los servicios públicos". Revista de Economía Institucional, 13, ISSN 0124-5996, Bogotá, pp 245-263 <http://www.scielo.org.co/pdf/rei/v7n13/v7n13a10.pdf>

(2011) CABALLERO MIGUEZ, Gonzalo, "Economía de las instituciones: de Coase y North a Williamson y Ostrom", Ekonomiaz, 77 ISSN 0213-3865, Vitoria, pp 14-51 <http://www1.euskadi.net/ekonomiaz/downloadPDF.apl?REG=1144>

(1984) COASE, Ronald, "The New Institutional Economics", Journal of Theoretical and Institutional Economics, 140(1), ISSN 1614-0559, Berlin, pp 229-231 $<$ http://www.jstor.org/stable/40750690>

(2001) DEL MONTE, Alfredo y PAGAGNI, Erasmo, "Public expenditure, corruption, and economic growth: the case of Italy", European Journal of Political Economy, 17 (1), ISSN 01762680, Amsterdam, pp 1-16, <http://dx.doi.org/10.1016/S0176-2680(00)00025-2>

(2007) FISMAN, Raymond y MIGUEL, Edward, "Corruption, Norms, and Legal Enforcement: Evidence from Diplomatic Parking Tickets", Journal of Political Economy, 115(6), ISSN 00223808, Chicago, pp 1020-1048, <http://www.jstor.org/stable/10.1086/527495>

(2008) HODGSON, Geoffrey y JIANG, Shuxia, "La economía de la corrupción y la corrupción de la economía: una perspectiva institucionalista", Revista de Economía Institucional, 18, ISSN 0124-5996, Bogotá, pp 55-80 <http://www.scielo.org.co/pdf/rei/v10n18/v10n18a3.pdf>

(2001) JAIN, Arvind K., "Corruption: A Review", Journal of Economic Surveys, 15(1), ISSN 1467-6419, pp 71-120, <http://darp.Ise.ac.uk/PapersDB/Jain_(JES01).pdf>

(1995) MAURO, Paolo, "Corruption and Growth", Quarterly Journal of Economics, 110(3), ISSN 1531-4650, Cambridge, $\mathrm{pp}$

681-712

<http://homepage.ntu.edu.tw/ kslin/macro2009/Mauro\%201995.pdf>

(1997) MAURO, Paolo, Why Worry about Corruption?, Economic Issues 6 Ed. International Monetary Fund, Washington, D.C.

(2003) NIETO, Alejandro, "La democracia corrompida", Cuadernos del Sureste, 11, Arrecife de Lanzarote $\mathrm{pp}$

92-103 <http://www.laopiniondelanzarote.com/cuadernos/pdfs/numero11/lademocraciacorrompida.pdf> (1991) NORTH, Douglass C., "Institutions", The Journal of Economic Perspectives, 5(1), ISSN 0895-3309, Pittsburgh, pp 97-112 $<$ http://www.econ.uchile.cl/uploads/documento/94ced618aa1aa4d59bf48a17b1c7f605cc9ace73 .pdf>

(2003) PARADA, Jairo J., "Economía Institucional Original y Nueva Economía Institucional: semejanzas y diferencias", Revista de Economía Institucional, 8, ISSN 0124-5996, Bogotá, pp 92-116 <http://www.scielo.org.co/pdf/rei/v5n8/v5n8a5.pdf>

(1996) ROSE-ACKERMAN, Susan, "¿Una administración reducida significa una administración más limpia?", Nueva sociedad, 145, ISSN 0251-3552, Buenos Aires, pp 66-79 <http://www.nuso.org/upload/articulos/2533_1.pdf>

(2000) SAVAS, E. S., Privatization and Public-Private Partnerships, Ed. Seven Bridges Press, New York

(2002) TANZI, Vito y DAVOODI, Hamid, "Corruption, Growth and Public Finances". En: G. T. Abed y S. Gupta (eds.), Governance, Corruption, and Economic performance, Ed. International Monetary Fund, pp 1-27.

(2000) TORRES, Gerver, "The Tortuous Road to Privatization in Venezuela". En: M. H. Birch y J. Haar (eds.), The Impact of Privatization in the Americas, Ed. North-South Center Press, Miami, pp 115-1176

(2000) TREISMAN, Daniel, "The causes of corruption: a cross-national study", Journal of Public Economics, 76, ISSN 0047-2727, Amsterdam, pp 399-457 
<http://www.policyinnovations.org/ideas/policy_library/data/01437/_res/id\%3Dsa_File1/Treisma n_Causes_Corruption.pdf>

(2006) VARSAKELIS, Nikos C., "Education, political institutions and innovate activity: A crosscountry empirical investigation", Research Policy, 35, ISSN 0048-7333, Brighton, pp 1083-1090 $<$ http://dx.doi.org/10.1016/j.respol.2006.06.002>

\section{A NEXO}

\section{Tabla 5. Puntuación de los países en el Î́ndice de Control de la Corrupción (2011)}

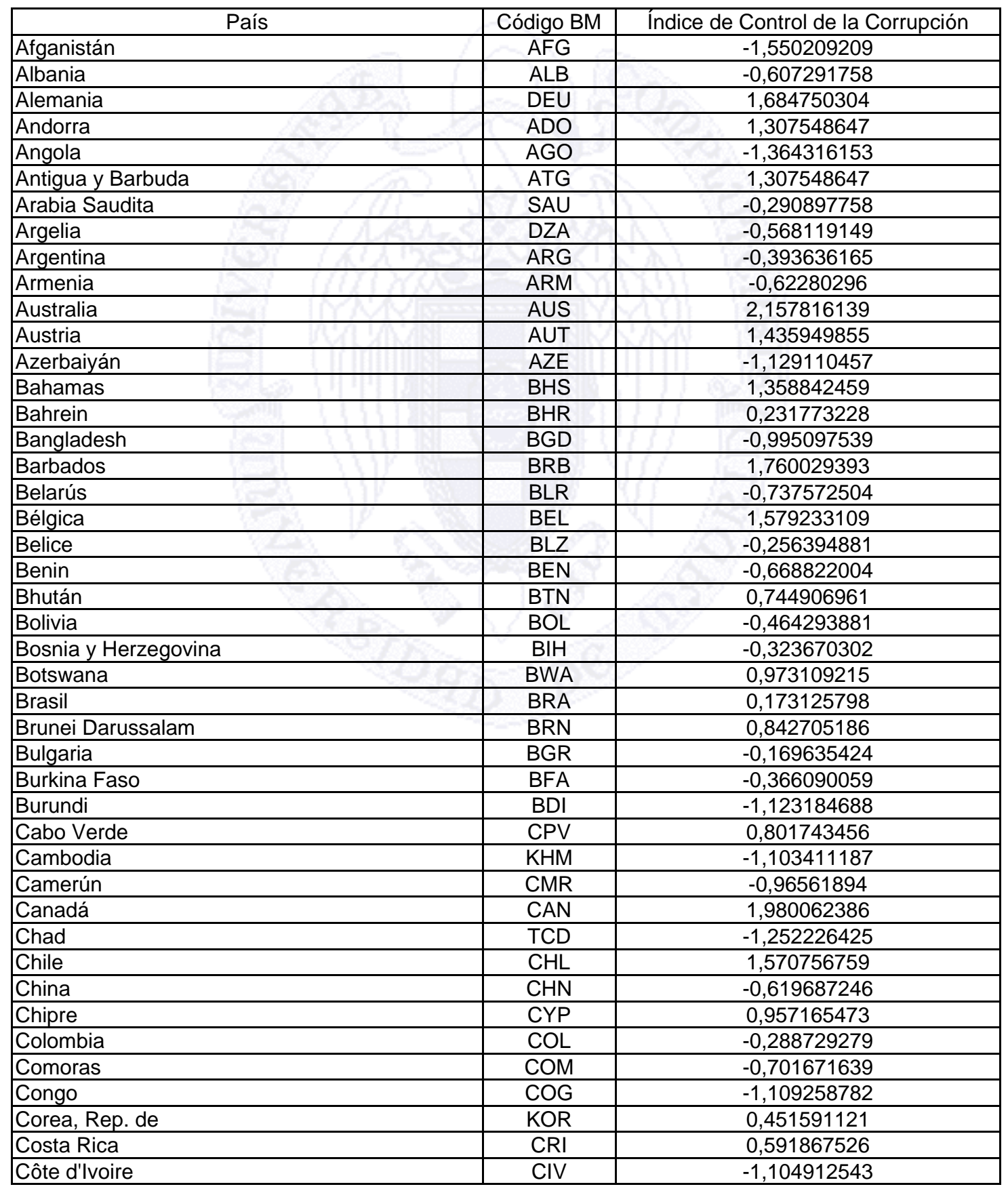




\begin{tabular}{|c|c|c|}
\hline Croacia & HRV & 0,023426371 \\
\hline Cuba & CUB & 0,442991208 \\
\hline Dinamarca & DNK & 2,421082664 \\
\hline Djibouti & DJI & $-0,298062245$ \\
\hline Dominica & DMA & 0,741865869 \\
\hline Ecuador & ECU & $-0,820904585$ \\
\hline Egipto & EGY & $-0,676259291$ \\
\hline El Salvador & SLV & $-0,22652587$ \\
\hline Emiratos Árabes Unidos & ARE & 1,084988605 \\
\hline Eritrea & ERI & $-0,552816824$ \\
\hline Eslovaquia & SVK & 0,292797075 \\
\hline Eslovenia & SVN & 0,930193571 \\
\hline España & ESP & 1,056759287 \\
\hline Estado de Palestina & WBG & $-0,829222807$ \\
\hline \begin{tabular}{|l|} 
Estados Unidos \\
\end{tabular} & USA & 1,233947189 \\
\hline Estonia & EST & 0,912103541 \\
\hline Etiopía & ETH & $-0,694452641$ \\
\hline Fiji & $\mathrm{FJI}$ & $-0,489360663$ \\
\hline Filipinas & $\mathrm{PHL}$ & $-0,784067547$ \\
\hline Finlandia & FIN & 2,185028233 \\
\hline Francia & FRA & 1,511963556 \\
\hline Gabón & GAB & $-0,767834731$ \\
\hline Gambia & GMB & $-0,498601092$ \\
\hline Georgia & GEO & $-0,038203157$ \\
\hline Ghana & $\mathrm{GHA}$ & 0,17131654 \\
\hline Granada & GRD & 0,440254723 \\
\hline 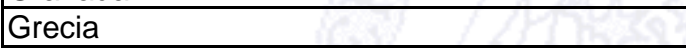 & GRC & $-0,145405349$ \\
\hline Guatemala & GTM & $-0,517724569$ \\
\hline Guinea & GIN & $-1,168406427$ \\
\hline Guinea Ecuatorial & GNQ & $-1,487105361$ \\
\hline Guinea-Bissau & GNB & $-1,058201484$ \\
\hline Guyana & GUY & $-0,601318183$ \\
\hline Haití & $\mathrm{HTI}$ & $-1,269006743$ \\
\hline Honduras & HND & $-0,801652606$ \\
\hline Hong Kong, China (RAE) & HKG & 1,838759076 \\
\hline Hungría & HUN & 0,341759273 \\
\hline India & IND & $-0,562417976$ \\
\hline Indonesia & IDN & $-0,655651407$ \\
\hline Irán, República Islámica del & IRN & $-0,914851301$ \\
\hline 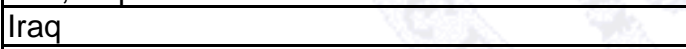 & IRQ & $-1,224688456$ \\
\hline 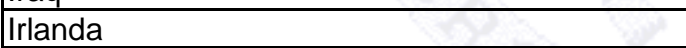 & $\mathrm{IRL}$ & 1,518482229 \\
\hline Islandia & ISL & 1,936775501 \\
\hline Islas Salomón & SLB & $-0,442089877$ \\
\hline Israel & ISR & 0,678023375 \\
\hline Italia & ITA & $-0,006872571$ \\
\hline Jamaica & JAM & $-0,376912239$ \\
\hline Japón & JPN & 1,50096503 \\
\hline Jordania & JOR & 0,041140289 \\
\hline Kazajstán & KAZ & $-1,012068701$ \\
\hline Kenya & KEN & $-0,869106964$ \\
\hline Kirguistán & KGZ & $-1,126280855$ \\
\hline Kiribati & KIR & 0,134077817 \\
\hline Kuwait & KWT & 0,074004892 \\
\hline Lao, República Democrática Popular & LAO & $-1,063398205$ \\
\hline \begin{tabular}{|l|} 
Lesotho \\
\end{tabular} & LSO & 0,224286554 \\
\hline Letonia & LVA & 0,207420663 \\
\hline Líbano & LBN & $-0,914191884$ \\
\hline Liberia & LBR & $-0,461189261$ \\
\hline Libia & LBY & $-1,308261309$ \\
\hline \begin{tabular}{|l} 
Liechtenstein \\
\end{tabular} & LIE & 1,812830215 \\
\hline Lituania & LTU & 0,286270882 \\
\hline Luxemburgo & LUX & 2,173120859 \\
\hline Macedonia (ERY) & MKD & $-0,038008042$ \\
\hline
\end{tabular}




\begin{tabular}{|c|c|c|}
\hline Madagascar & MDG & $-0,278178919$ \\
\hline Malasia & MYS & 0,003221345 \\
\hline Malawi & $\mathrm{MWI}$ & $-0,36221119$ \\
\hline Maldivas & MDV & $-0,625521575$ \\
\hline Malí & $\mathrm{MLI}$ & $-0,613258585$ \\
\hline Malta & MLT & 0,906341027 \\
\hline Marruecos & MAR & $-0,260241846$ \\
\hline Mauricio & MUS & 0,618535656 \\
\hline Mauritania & MRT & $-0,569350462$ \\
\hline México & MEX & $-0,363141852$ \\
\hline Micronesia, Estados Federados de & FSM & $-0,300714312$ \\
\hline Moldova, Rep. de & MDA & $-0,617298399$ \\
\hline Mongolia & MNG & $-0,665095865$ \\
\hline Montenegro & MNE & $-0,211581582$ \\
\hline Mozambique & $\mathrm{MOZ}$ & $-0,407113823$ \\
\hline Myanmar & MMR & $-1,685367264$ \\
\hline Namibia & NAM & 0,222526293 \\
\hline Nepal & NPL & $-0,769821579$ \\
\hline Nicaragua & $\mathrm{NIC}$ & $-0,766122663$ \\
\hline Níger & NER & $-0,645933239$ \\
\hline Nigeria & NGA & $-1,137404342$ \\
\hline Noruega & NOR & 2,168954338 \\
\hline Nueva Zelandia & NZL & 2,330007554 \\
\hline Omán & OMN & 0,077472089 \\
\hline Países Bajos & NLD & 2,169215039 \\
\hline Pakistán & PAK & $-1,002826152$ \\
\hline 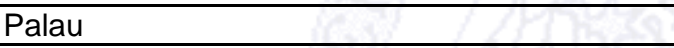 & PLW & $-0,460629059$ \\
\hline Panamá & PAN & $-0,353312956$ \\
\hline Papua Nueva Guinea & PNG & $-1,121928445$ \\
\hline Paraguay & PRY & $-0,730197325$ \\
\hline Perú & PER & $-0,204246121$ \\
\hline Polonia & $\mathrm{POL}$ & 0,514028256 \\
\hline Portugal & PRT & 1,088102276 \\
\hline Qatar & QAT & 1,024691032 \\
\hline Reino Unido & GBR & 1,536946982 \\
\hline República Centroafricana & CAF & $-0,84646901$ \\
\hline República Checa & CZE & 0,315825723 \\
\hline República Democrática del Congo & ZAR & $-1,371275213$ \\
\hline República Dominicana & DOM & $-0,790515019$ \\
\hline+2 & ROM & $-0,200344908$ \\
\hline Rusia, Federación de & RUS & $-1,092239746$ \\
\hline+2 & RWA & 0,448868493 \\
\hline Saint Kitts y Nevis & KNA & 1,059461104 \\
\hline Samoa (Occidental) & WSM & 0,110170611 \\
\hline San Vicente y las Granadinas & VCT & 1,059461104 \\
\hline Santa Lucía & LCA & 1,235878748 \\
\hline Santo Tomé y Príncipe & STP & $-0,368725068$ \\
\hline Senegal & SEN & $-0,615088471$ \\
\hline Serbia & SRB & $-0,197428855$ \\
\hline Seychelles & SYC & 0,258106227 \\
\hline Sierra Leona & SLE & $-0,694087502$ \\
\hline Singapur & SGP & 2,11793007 \\
\hline Siria, República Árabe & SYR & $-0,974855592$ \\
\hline Sri Lanka & LKA & $-0,423418548$ \\
\hline Sudáfrica & ZAF & 0,034038951 \\
\hline Sudán & SSD & $-1,647649442$ \\
\hline Suecia & SWE & 2,22063993 \\
\hline Suiza & $\mathrm{CHE}$ & 2,019146176 \\
\hline Suriname & SUR & $-0,364165147$ \\
\hline Swazilandia & SWZ & $-0,265234548$ \\
\hline Tailandia & THA & $-0,36664706$ \\
\hline Tanzania, República Unida de & TZA & $-0,523604448$ \\
\hline Tayikistán & TJK & $-1,134037125$ \\
\hline
\end{tabular}




\begin{tabular}{|l|c|c|}
\hline Timor-Leste & TMP & $-1,050617495$ \\
\hline Togo & TGO & $-0,992423185$ \\
\hline Tonga & TON & $-0,292399156$ \\
\hline Trinidad y Tobago & TTO & $-0,275726173$ \\
\hline Túnez & TUN & $-0,210853757$ \\
\hline Turkmenistán & TKM & $-1,455338583$ \\
\hline Turquía & TUR & 0,099556684 \\
\hline Ucrania & UKR & $-0,990256663$ \\
\hline Uganda & UGA & $-0,861736499$ \\
\hline Uruguay & URY & 1,282242286 \\
\hline Uzbekistán & UZB & $-1,338881954$ \\
\hline Vanuatu & VUT & 0,348740178 \\
\hline Venezuela, República Bolivariana de & VEN & $-1,21513589$ \\
\hline Viet Nam & VNM & $-0,593297717$ \\
\hline Yemen & YEM & $-1,1803772$ \\
\hline Zambia & ZMB & $-0,514948361$ \\
\hline Zimbabwe & ZWE & $-1,297314165$ \\
\hline
\end{tabular}

FUENTE: Indicadores de Gobernabilidad (Banco Mundial) http://info.worldbank.org/governance/wgi/index.asp 